\title{
MNB1 gene is involved in regulating the iron- deficiency stress response in Arabidopsis thaliana
}

\section{Hui Song}

Hefei University of Technology

\section{Feng Chen}

Hefei University of Technology

Xi Wu

Hefei University of Technology

Min $\mathrm{Hu}$

Hefei University of Technology

Qingliu Geng

Hefei University of Technology

Min Ye

Hefei University of Technology

Cheng Zhang

Hefei University of Technology

Li Jiang

Hefei University of Technology

Shuqing Cao ( $\nabla$ shuqingcao@hfut.edu.cn)

Hefei University of Technology https://orcid.org/0000-0001-6693-877X

\section{Research Article}

Keywords: Fe-deficiency, MNB1, Reactive oxygen species, Arabidopsis

Posted Date: September 8th, 2021

DOI: https://doi.org/10.21203/rs.3.rs-833563/v1

License: (c) (i) This work is licensed under a Creative Commons Attribution 4.0 International License.

Read Full License 
$1 \quad M N B 1$ gene is involved in regulating the iron-deficiency stress

2 response in Arabidopsis thaliana

3 Hui Song ${ }^{1,2^{*}}$, Feng Chen ${ }^{1,2^{*}}, \mathrm{Xi} \mathrm{Wu}^{1,2^{*}}$, Min $\mathrm{Hu}^{1,2}$, Qingliu Geng ${ }^{1,2}$, Min Ye ${ }^{1,2}$, Cheng

4 Zhang $^{1,2}$, Li Jiang ${ }^{1,2 \S}$ and Shuqing $\mathrm{Cao}^{1,2 \S}$

$5 \quad{ }^{1}$ Engineering Research Center of Bio-process, Ministry of Education, ${ }^{2}$ School of Food

6 and Biological Engineering, Hefei University of Technology, Hefei 230009, China.

7

$8 \quad *$ These authors contributed equally to this work.

9 §Corresponding author: shuqingcao@hfut.edu.cn or jiangli@ustc.edu.cn

10

11

12 
Key message MNB1 gene negatively controls the Fe-deficiency response in Arabidopsis via modulating ROS levels and the ROS-mediated signaling pathway, thereby affecting the expression of Fe-uptake and translocation genes

Abstract Iron (Fe) is an indispensable mineral element for normal growth of plants. Fe deficiency induces a complex series of responses in plants, involving physiological and developmental changes, to increase Fe uptake from soil. However, the molecular mechanism involved in plant Fe-deficiency is not well understood. Here, we found that the MNB1 gene is involved in modulating Fe-deficiency response in Arabidopsis thaliana. The expression of MNB1 was inhabited by Fe-deficiency stress. Knockout of MNB1 led to enhanced Fe accumulation and tolerance, whereas the MNB1overexpressing plants were sensitive to Fe-deficiency stress. Lower $\mathrm{H}_{2} \mathrm{O}_{2}$ concentrations in mnbl mutant plants were examined under Fe deficiency circumstances compared to wild-type. On the contray, higher $\mathrm{H}_{2} \mathrm{O}_{2}$ concentrations were found in MNB1-overexpressing plants, which was adversely linked with malondialdehyde (MDA) concentrations. Furthermore, in mnbl mutants, the transcription level of the Fe-uptake and translocation genes, FIT, IRT1, FRO2, ZIF, FRD3, NAS4, PYE and MYB72, were considerably elevated during Fe-deficiency stress, resulting in higher Fe accumulation. Together, our findings show that the MNB1 gene negatively controls the Fe-deficiency response in Arabidopsis via modulating reactive oxygen species (ROS) levels and the ROS-mediated signaling pathway, thereby affecting the expression of Fe-uptake and translocation genes.

Key words: Fe-deficiency $\cdot M N B 1 \cdot$ Reactive oxygen species $\cdot$ Arabidopsis

\section{Introduction}

Iron $(\mathrm{Fe})$ is a micronutrient that is required for plant metabolism, growth, and development. It functions as a cofactor for metalloproteins, which are involved in a variety of biological processes. (Hansch and Mendel., 2009; Balk and Schaedler., 2014). Although $\mathrm{Fe}$ is a plentiful element in the environment, it is frequently found in salinealkaline and calcareous soils as insoluble ferric hydroxides, resulting in low Fe 
bioavailability for plants (Xiao et al., 2015). As a result, understanding the molecular mechanisms underpinning Fe absorption and trafficking is crucial for improving $\mathrm{Fe}$ bioavailability and content in plants. To counteract Fe acquisition from soil, plants have evolved two key mechanisms to absorb Fe: (1) the reduction-based strategy (Strategy I) mechanism found in all dicots and non-graminaceous monocots, and (2) the chelation-based strategy (Strategy II) mechanism found only in graminaceous plants. (Jeong and Guerinot., 2009; Walker and Connolly., 2008; Hindt and Guerinot., 2012). The strategy I mechanism involves protons being extruded to lower the $\mathrm{pH}$ of the rhizosphere, allowing FRO2 (Ferric reductase oxidase 2) to convert ferric iron compounds to more soluble $\mathrm{Fe}^{2+}$, and then transporting ferrous Fe from the soil into the root epidermal cell membrane via IRT1 (Fe-regulated transporter 1; Eide et al., 1996; Vert et al., 2002; Hell and Stephan., 2003); whereas under low Fe stress, the phytosiderophores (mugineic acids) released by strategy II graminaceous plants chelate ferric Fe, and the resultant complexes are delivered into root cells by the YS1 (Yellowstripe 1 transporters; Curie et al., 2001; Kobayashi et al., 2010). In addition, plants have evolved a number of regulatory mechanisms at both the transcriptional and posttranscriptional levels to maintain Fe homeostasis, owing to the crucial biological activities of Fe. Several transcription factors have been verified to modulate $\mathrm{Fe}$ deficiency responses in Arabidopsis, including FIT, bHLH38, bHLH39, bHLH100, bHLH101, bHLH34, bHLH104, bHLH105, bHLH115, PYE, MYB10 and MYB72 et al (Ling et al., 2002; Colangelo and Guerinot., 2004; Jakoby et al., 2004; Yuan et al., 2008; Long et al., 2010; Palmer et al., 2013; Wang et al., 2013; Zhang et al., 2015; Li et al., 2016b; Liang et al.,2017; Cui et al., 2018). These findings aid our understanding of plant responses to Fe shortage stress, although a number of novel genes involved in iron homeostasis still need to be identified. According to new research, Carbohydratebinding proteins, commonly known as agglutinins or lectins, are found in a wide range of plant species and have an important biological function in pathogen defense responses (Peumans and Van Damme., 1995). Plant mannose binding lectins detect specific protein-carbohydrate combinations on pathogen surfaces and serve a key role in plant defense mechanisms against pathogens (Hwang and Hwang., 2011; Van 
Damme et al., 2004). Because of an interaction with cell wall extracellular glycans or carbohydrates, several plant lectins are vital for bacterial defense via an indirect biochemical process. All contemporary plant lectins have been grouped into twelve plant lectin clusters of small and structurally similar proteins, according to earlier research (Van Damme et al., 2008). The pepper mannose-binding lectin (CaMBL1) was recently discovered to play a role in microbial pathogen defense, and mannose has been confirmed to bind to CaMBL1(Hwang and Hwang., 2011). Mannose is thought to have a major role in plant resistance to cadmium toxicity, according to a recent study (Chen et al., 2015). Also, we found that MNB1, an Arabidopsis homolog of CaMBL1, also modulates Cd tolerance (Yan et al., 2021). However, whether MNB1 is involved in the modulation of Fe-deficiency stress is uncertain.

In the present study, we showed that the $M N B 1$ gene negatively modulates Fedeficiency response. The transcription of the $M N B 1$ gene was inhibited by Fedeficiency stress, and the mnbl mutants showed increased Fe-deficiency tolerance. The MNB1 gene generated reactive oxygen species (ROS)-mediated signaling in response to Fe shortage stress by controlling ROS levels, which affected the expression of Feuptake and translocation genes, resulting in increased Fe accumulation and tolerance.

\section{Materials and methods}

\section{Plant materials, growth conditions, and treatments}

In this work, the A. thaliana wild-type (Col-0), mnb1 mutants (mnb1-1: SALK038821C; mnb1-2: SALK-121641; ecotypes Columbia), and the transgenic lines overexpressing MNB1 were used as plant materials (Alonso et al., 2003; Yan et al., 2021). Arabidopsis seeds were surface-sterilized in $0.1 \mathrm{M} \mathrm{HgCl}_{2}$, and washed three times with sterile $\mathrm{ddH}_{2} \mathrm{O}$ water to remove $0.1 \mathrm{M} \mathrm{HgCl}_{2}$ solution, and then germinated on Murashige and Skoog (MS media, Caisson, USA) nutrient medium, supplemented with $1 \%$ sucrose $(\mathrm{w} / \mathrm{v})$ and $1.2 \%(\mathrm{w} / \mathrm{v})$ agar, adjusted to $\mathrm{pH}$ 5.8. Culture plates were vernalized for 3 days in a refrigerator in the dark at $4{ }^{\circ} \mathrm{C}$ and then placed into a culture chamber set at $22{ }^{\circ} \mathrm{C}$, under a light intensity of $100 \mu \mathrm{mol} \mathrm{m} \mathrm{m}^{-2} \mathrm{~s}^{-1}$ and a long day $(16 \mathrm{~h}$ of light/8 h of dark cycles). 
For phenotypic analyses of the Fe-deficiency response, seeds of Col, mnb1-1, mnb1-2, OE3, and OE7 (Yan et al., 2021) plants were germinated on MS media for 3 days and then transferred to $+\mathrm{Fe}$ [MS media as a control] or Fe-deficiency medium [Fe, without Fe(II)-EDTA; -Fe+Frz, with $50 \mathrm{mM}$ ferrous chelate ferrozine (Sangon Biotech, China)]. The plants were photographed after two weeks of growth and then submitted to growth experiments, with their root length and total chlorophyll content measured. All experiments were carried out three times independently, with over 30 Arabidopsis seedlings used in each measurement. For Fe-deficient inducible gene expression analysis, the Arabidopsis seedlings were grown for 10 days on MS medium and then transferred to $\mathrm{MS}(+\mathrm{Fe})$ or $-\mathrm{Fe}$ medium for 7 days, finally, these plant materials were applied for further analysis.

\section{Generation of 35S:MNB1-GFP transgenic plants}

116 To generate 35S:MNB1-GFP (green fluorescent protein) transgenic plants, the CDS 117 region of $M N B 1(\mathrm{AT} 1 \mathrm{~g} 78830$ ) was amplified from Arabidopsis with specific primers by 118 PCR (Table S1), digested with Kpn1 and Xho1 restriction enzymes (called 35S:MNB1119 GFP), and then cloned into the $p A R T 27$ vector containing Cauliflower mosaic virus 35S promoter and GFP reporter. The 35S:MNB1-GFP recombinant vectors were introduced into the Agrobacterium GV3101 strain and then transformed into Arabidopsis wild-type lines by using the floral dip method (Clough et al., 1998). The 35S:MNB1-GFP lines were T3 homozygous plants used in this work. All the obtained transgenic lines were chosen for further experiments.

\section{RNA extraction and real-time quantitative RT-PCR (qRT-PCR) analysis}

Total RNA of whole seedlings or different tissues was extracted using Trizol Reagent (Invitrogen, USA) following the manufacturer's instructions and then used to synthesize cDNA. Reverse transcription reactions were performed as described (Fan et al., 2016). Real-time quantitative RT-PCR(qRT-PCR) was conducted in the Bio-Rad iCycler iQ system (Bio-Rad Laboratories, USA) applying a Platinum SYBR Green qPCR SuperMix-UDG (Invitrogen, USA) following the manufacturer's method. 
ACTIN8 (AT1G49240) was used as the internal control. All experiments were carried out at least in triplicate. The specific qRT-PCR primers used are listed in Supplementary Table S1.

\section{3,3'-Diaminobenzidine (DAB) staining}

DAB staining was performed according to an adaptation of a previously reported protocol (Nakagami et al., 2006). Specifically, Arabidopsis seedling samples were vacuum-immersed in 3,3'-diaminobenzidine solution (DAB, $1 \mathrm{mg} / \mathrm{mL}, \mathrm{pH}$ 3.8, SigmaAldrich) for 15 minutes before being incubated in a gyratory shaker at $25^{\circ} \mathrm{C}$ for 3-4 hours. Following the completion of the DAB staining reaction, these samples were fixed in a bleaching solution (ethanol/acetic acid/glycerol $=3: 1: 1$ ). After that, using a camera and ImageJ software, samples were photographed and quantified (Ruifrok and Johnston 2001).

\section{Protein extraction and western blotting assay}

Total protein of different Arabidopsis samples was extracted and boiled as previously described (Ren et al., 2018). Briefly, Proteins were separated by $10 \%$ sulfatepolyacrylamide gel electrophoresis (SDS-PAGE). After transferring to poly (vinylidene fluoride) (PVDF) membranes and blocking with 4\% nonfat milk, immunoblot was probed using specific antibodies. The plant antibodies were purchased from the PhytoAB company (California, USA). Western Blotting Detection System was conducted using an EasySee Western Blot Kit (TransGen Biotech, China). To analyze the protein expression, Rubisco was used as a control.

\section{Malondialdehyde (MDA) measurement.}

Determination of malondialdehyde (MDA) content in Arabidopsis seedlings through the thiobarbituric acid reaction as used in the method described by Hodges et al., (1999). Wild-type, mnb1-1, mnb1-2, OE3, and OE7 Arabidopsis seedling samples were taken and homogenized in 80 percent (v/v) ethanol. The homogenate was centrifuged for 10 minutes at $11,500 \mathrm{~g}$, and the supernatant was aliquoted and mixed with $1.5 \mathrm{~mL} 5 \%$ 
$(\mathrm{w} / \mathrm{v})$ trichloroacetic acid containing varying amounts of thiobarbituric acid. The reaction mixture was then heated at $95^{\circ} \mathrm{C}$ for $30 \mathrm{~min}$ in a water bath and then rapidly cooled in an ice bath. The spectrophotometric absorbance was monitored at 450, 532, and $600 \mathrm{~nm}$. The concentration of MDA was calculated following parameters of various dilutions of reference solutions.

\section{Ferric Chelate Reductase (FCR) Assays}

The measurement of FCR activity was carried out according to a previous study (Yi and Guerinot., 1996). Briefly, fresh 10 whole seedlings of each sample were pretreated for 30 minutes in glass plates with $4 \mathrm{~mL}$ MS solution absence micronutrients at $\mathrm{pH} 5.5$ ( $\mathrm{pH}$ adjusted by addition of $\mathrm{HCl}$ ) and then immersed for 40 minutes in the dark with 5 $\mathrm{mL} \mathrm{Fe}$ (III) reduction assay solution [MS solution absence micronutrients, $0.3 \mathrm{mM}$ ferrozine, and $0.1 \mathrm{mM} \mathrm{Fe}$ (III)-EDTA (at pH 5.0)].

\section{Determination of total chlorophyll content}

Total chlorophyll was extracted from two-week-old seedlings in darkness at room temperature using 80 percent acetone. At $645 \mathrm{~nm}$ and $663 \mathrm{~nm}$, the supernatant was spectrophotometrically analyzed. The total chlorophyll content was determined by modifying the protocol of Aono et al., (1993).

\section{Fe Concentration Measurement}

For Fe Concentration Measurement, Arabidopsis seeds were grown on the Fe-sufficient or Fe-deficient medium for two weeks. The shoots and roots were collected separately and used for the analysis of Fe content measurement. Tissue samples were dried at $100{ }^{\circ} \mathrm{C}$ for $30 \mathrm{~min}$ and $80{ }^{\circ} \mathrm{C}$ for 2 days, weighed, and digested in a mixture of concentrated nitric acid/ perchloric acid (3:1) in a microwave digestion system ETHOS1 (Milestone). Three samples were used for Fe content measurement in each independent experiment, and $\mathrm{Fe}$ concentrations were measured by ICPOES (model 5300DV; PerkinElmer, USA), as described previously (Herbik et al., 2002). 


\section{Results}

The mnb1 mutants showed enhanced Fe-deficiency stress tolerance

We studied the reaction of loss-of-function mnbl mutants (Yan et al., 2021) to Fedeficiency stress to see if MNB1 is involved in the regulation of Fe-deficiency tolerance. We discovered that when the Col and mnbl mutant plants were cultivated on MS medium, there was no significant difference between them; whereas mnbl mutant plants demonstrated remarkable tolerance when grown on Fe-deficient medium (-Fe, without $\mathrm{Fe}$; -Fe+Frz, with $50 \mathrm{mM}$ ferrozine to chelate micro metal of Fe from agar), with longer root length and greener leaves than Col (Fig. 1A). Under Fe-limited circumstances $(P<0.05$; Fig. $1 \mathrm{~B}, \mathrm{C})$, the mnbl mutant plants' root length and total chlorophyll content were considerably higher than the Col.

\section{MNB1-overexpressing plants exhibited increased sensitivity to Fe deficiency}

Analyzing the phenotypes of MNB1-overexpressing transgenic plants validated the role of MNB1 in Fe-deficiency stress response. In comparison to Col, the MNB1overexpressing transgenic plants OE3 and OE7(Yan et al., 2021) displayed considerable reduction of root growth and chlorotic cotyledons when cultivated on Fe-deficient (-Fe, -Fe+Frz) conditions (Fig. 2A). The development of the Col, OE3 and OE7 lines in MS medium was similar (Fig. 2A). Under Fe-limited circumstances, the root length and total chlorophyll content of MNB1-overexpressing transgenic plants were lower than that of $\mathrm{Col}(P<0.05$; Fig. 2B, C). These findings suggest that MNB1 is important in the control of the Fe-deficiency stress response.

\section{Expression of MNB1 is repressed by Fe-deficiency stress}

To evaluate the expression profiles of $M N B 1$ in Fe-deficiency stress response, the Col seedlings were harvested after treatment for 7 days under conditions of MS and $\mathrm{Fe}-$ deficient, and the expression abundance of FIT and MNB1 was analyzed by quantitative real-time PCR. In response to Fe-deficiency stress, the expression of FIT was significantly induced $(P<0.05$; Fig. 3A), whereas the expression of $M N B 1$ significantly decreased $(P<0.05$; Fig. 3B). In addition, MNB1-GFP transgenic plants were further 
produced to determine the protein levels of MNB1 in response to Fe-deficiency stress. Western blot results showed that the MNB1 protein accumulation decreased under Fedeficient conditions (Fig. 3C). These results further confirmed that MNB1 is involved in the regulation of Fe-deficiency stress response.

\section{Loss-of-function of MNB1 reduces ROS level in response to Fe-deficiency stress} It was previously indicated that knockout of the CaMBL1 gene resulted in increased disease susceptibility, enhanced bacterial growth, reduced production of ROS in response to infection with virulent or avirulent $\mathrm{Xcv}$ in pepper leaves (Hwang and Hwang., 2011). Thus, we hypothesized that the loss-of-function of MNB1 decreased the accumulation of ROS in response to Fe-deficiency stress. We utilized DAB staining to examine changes in ROS levels in mnbl mutants and MNB1-overexpressing plants under normal and Fe-deficiency stress conditions to test this theory. Under Fedeficiency stress conditions, the $\mathrm{H}_{2} \mathrm{O}_{2}$ concentrations of the mnb1 mutants, MNB1overexpressing, and wild-type plants differed. When $m n b l$ mutants were compared to $\mathrm{Col}$, the $\mathrm{H}_{2} \mathrm{O}_{2}$ level was lower in mnb1 mutants, but greater in MNB1-overexpressing plants. (Fig. 4A; $P<0.05$, Fig. 4B).

Due to the fact that ROS leads to cellular damage in vivo (Suzuki et al., 2012), we detected the MDA level in Col, mnb1-1, mnb1-2, OE3, OE7 plants under normal and Fe-deficiency conditions. We observed that MDA content was lower in the mnbl mutant plants and greater in the MNB1-overexpressing lines under Fe deficiency stress compared to $\mathrm{Col}(P<0.05$, Fig. 4C). This revealed a relationship between ROS levels and MDA levels (Fig. 4A, C). The above results indicate that mnbl mutant plants encountered reduced oxidative damage under Fe-deficiency stress conditions.

Loss-of-function of MNB1 led to enhanced expression of genes related to Fedeficiency under Fe-limited conditions

The above experimental results indicated that The MNB1 gene influenced ROS levels and may have produced ROS-mediated signaling, as well as influencing the expression 
abundances of Fe-related genes in plants in response to Fe-deficiency stress. To see if MNB1 had an effect on Fe-related gene expression, we compared the relative expression of Arabidopsis genes involved in Fe absorption and translocation in Col and mnb1 lines. Under Fe-deficiency stress, transcription levels of several key Fe uptake and translocation genes were examined, including FIT, IRT1, FRO2, ZIF1, FRD3, NAS4, PYE and MYB72 (Ling et al., 2002; Colangelo and Guerinot, 2004; Jakoby et al., 2004; Long et al., 2010; Liang et al.,2017; Cui et al., 2018), and we found the expression of FIT, IRT1, FRO2, ZIF1, FRD3, NAS4, PYE and MYB72 were significantly higher in the mnb1 mutants in comparison with $\mathrm{Col}(P<0.05$; Fig. 5). In addition, the protein level of IRT1 in mnb1 mutants was further analyzed in response to Fe-deficiency stress. The buildup of IRT1 protein increased under Fe-deficient (-Fe) conditions, according to Western blot data (Fig. 5I). These findings show that MNB1-mediated ROS signaling promotes the expression of $\mathrm{Fe}$ uptake and translocation genes, resulting in greater $\mathrm{Fe}$ accumulation and improved Fe deficiency tolerance.

\section{Loss-of-function of MNB1 increases FCR activity.}

Previous studies have shown that FCR activity is also a typical indicator of physiology when the Fe is limited, thus we analyzed FCR activity of Col, mnbl mutants, and MNB1-overexpressing lines using the ferrozine assay under normal and Fe-deficiency stress conditions (Yi and Guerinot.,1996). We discovered no significant difference in FCR activity between Col, mnb1 mutants, and MNB1-overexpressing plants under normal circumstances. However, under Fe-deficiency stress conditions, the FCR activity of the mnbl mutants was significantly higher than that of Col, while the FCR activity of the MNB1-overexpressing lines was significantly lower than that of Col $(P<0.05$; Fig. 6A, B). Taken together, these results indicate that MNB1-mediated Fedeficiency tolerance is associated with increased FCR activity.

Loss-of-function of MNB1 led to increased Fe concentration level in response to Fe-deficiency stress

$F I T, I R T 1$, and FRO2 genes play a vital role in iron acquisition in plants (Yuan et al., 
2005; Bauer et al.,2007). The above results showed that the expression level of Fe uptake and translocation genes, including FIT, IRT1, FRO2, ZIF1, FRD3, NAS4, PYE, and MYB72 increased in the mnbl mutants than Col under Fe-deficiency stress. Therefore, we measured the Fe concentration of Col, mnbl mutants, and MNB1overexpressing plants under normal and Fe-deficiency conditions. Under Fe-deficiency stress conditions, we found that the Fe content was significantly higher in the leaves and roots of the mnb1 mutants compared with that $\mathrm{Col}(P<0.05$; Fig. $7 \mathrm{~A}, \mathrm{~B})$, while the Fe content in the leaves and roots of the OE3 and OE7 plants was significantly lower than that $\mathrm{Col}(P<0.05$; Fig. $7 \mathrm{C}, \mathrm{D})$. Taken together, these results suggest that MNB1mediated $\mathrm{Fe}$ accumulation and tolerance were consistent with increased expression of Fe-uptake genes (Fig. 5).

\section{Discussion}

$\mathrm{Fe}$ is an indispensable mineral element for the normal growth of plants. Fe shortage leads to delayed growth and decreased photosynthesis, resulting in lower crop production. To cope with the threat of a Fe-limited environment, plants may sense external Fe status and use complex systems to adjust the expression of Fe uptakerelated genes, allowing Fe to influx from soils to meet the plant's needs. Iron, on the other hand, is harmful to plants in excess due to the generation of hydroxyl radicals via the Fenton reaction, which is the most active ROS (Toyokuni., 1996; Thomine and Vert., 2013). Thus, it is really key to maintain Fe homeostasis in plants. Plants have evolved complicated regulatory networks to modulate Fe homeostasis and deficiency responses. However, the molecular regulatory mechanisms for Fe-deficiency stress response are still not well understood in plants, and only a number of Fe deficiency-responsive genes have been identified and characterized (Ling et al., 2002; Colangelo and Guerinot., 2004; Jakoby et al., 2004; Long et al., 2010; Liang et al.,2017; Cui et al., 2018). In previous research, mannose-binding lectins play important roles in many biological processes of plants, including defense signaling response during pathogen attack, and plant hormone responses, by specifically binding to carbohydrates (Lis and Sharon., 1998; Van Damme et al., 2008; Hwang and Hwang., 2011; Han et al., 2018). It has been 
reported that the association of pepper mannose-binding lectin CaMBL1 with mannose may play a crucial role in modulating cell death and defense responses against microbial pathogens (Hwang and Hwang., 2011). To our knowledge, so far, whether plant mannose-binding lectins modulates Fe deficiency responses or not, and how it occurs, remain unknown. In the present study, we found that the Arabidopsis mannose-binding lectin MNB1 negatively modulates the $\mathrm{Fe}$ accumulation and tolerance under $\mathrm{Fe}$ deficient conditions. According to the evidence presented here, $M N B 1$ transcription was severely repressed, and MNB1 protein accumulation decreased in response to $\mathrm{Fe}$ shortage stress (Fig. 3B, C). Additionally, the loss-of-function of MNB1 displayed enhanced tolerance to Fe deficiency, including increased root length, chlorophyll content, and FCR activity (Fig. 1,6), whereas overexpression of MNB1 plants showed sensitivity phenotype to Fe deficiency, including decreased root length, FCR activity, and chlorotic leaves (Fig. 2,6). ROS homeostasis is widely recognized to promote cellular development and proliferation as a result of positive ROS signaling; nevertheless, excessive ROS production causes oxidative stress, which can lead to cellular damage or even death (Wen et al., 2011). The study by Sun et al., (2016) indicated that ROS mediates between positive and negative regulation of plant responses to Fe deficiency stress and ROS participates in Fe distribution in roots. Under Fe shortage stress, we found that the total Fe content of roots and leaves in both mnbl mutants and MNB1-OE lines was significantly different from Col (Fig. 7). MNB1 or ROS may be participated in Fe distribution between roots and shoots, based on this finding. Furthermore, previous research shows that silencing the pepper mannosebinding lectin increased disease susceptibility, increased bacterial growth, and reduced ROS production in response to a virulent or avirulent Xcv infection in pepper leaves. As a result, we hypothesized that in Arabidopsis, the analogous mannose-binding protein MNB1 would affect plant responses to Fe shortage stress via ROS-mediated signaling. MDA levels in Col, mnb1-1, mnb1-2, and MNB1-OE lines under treatments with or without Fe confirmed this notion concerning MNB1 participation in our investigation (Fig. 4 C). Surprisingly, we found that ROS level in mnbl mutants was lower than Col in Fe-deficiency treatments, whereas ROS level in MNB1- 
overexpressing plants was higher than Col in Fe-deficiency treatments (Fig. 4 A, B). These findings suggest that MNB1 may regulate the Fe-deficiency stress response by affecting the endogenous ROS level. ROS plays a critical part in the complex communication networks that activate defensive systems when a plant is exposed to biotic or abiotic stresses (Hammond-Kosack and Parker., 2003). Proteins embedded in plasma membranes have been discovered to be part of a monitoring system that is required for the identification and transduction of defense-related signals (Garcia-

Brugger et al.,2006). Recently, a study reported that MNB1 is a membrane-associated protein (Yan et al., 2021). Therefore, we speculated that MNB1 would exert an important role in signal transduction. However, the biochemical function of MNB1 needs further study in future research. Stress caused by a lack of Fe impacted MNB1 transcription, changing the quantity of ROS and ROS-mediated signaling, as a result, the expression of Fe-related genes in the nucleus. In response to Fe shortage stress, plants have evolved a number of adaptive molecular mechanisms, the most notable of which are the Fe absorption and translocation routes (Yuan et al., 2005; Bauer et al., 2007). According to earlier studies, ROS-mediated signaling is transduced to the nucleus, where it regulates Fe-deficiency tolerance by altering the expression of nuclear genes linked to Fe-deficiency stress (Long mei et al., 2018), such as FIT, IRT1, and FRO2.As a result, we looked at the transcription levels of genes linked to Fe deficiency stress. MNB1 silencing increased the expression patterns of FIT, IRT1, FRO2, ZIF, FRD3, NAS4, PYE and MYB72 during Fe deficient stress (Fig. 5). As a result, our findings revealed that the $M N B 1$ gene modulated Fe uptake and translocation genes, hence regulating Fe deficiency stress.

In the present work, we also investigated whether MNB1 is involved in modulating other abiotic stress responses, such as $\mathrm{MnSO}_{4}$, High Fe, and $\mathrm{H}_{2} \mathrm{O}_{2}$, and discovered that the growth of mnb1-1 and mnb1-2 mutants under MnSO4, High Fe, and $\mathrm{H}_{2} \mathrm{O}_{2}$ stresses was significantly different from that of Col, indicating that MNB1 may be involved in modulating $\mathrm{MnSO} 4$, High Fe, and $\mathrm{H}_{2} \mathrm{O}_{2}$ stress responses. Consequently, investigating the molecular mechanisms of MNB1-mediated MnSO4, High Fe, and H2O2 stresses could be fascinating (Fig. S1). Furthermore, because MNB1 proteins have been shown 
to bind to d-Mannose, we investigated whether the mannose is required for MNB1mediated Fe deficiency tolerance (Yan et al., 2021). To test this hypothesis, we employed exogenous mannose to deal with Col, mnb1-1, mnb1-1, OE3, and OE7 plants, and found that no notable differences were observed to exogenous mannose was added with Fe-limited media (Fig. S2). This may suggest that mannose is not required for MNB1-mediated Fe deficiency tolerance.

In conclusion, our findings shed light on the biological functions of mannosebinding lectins in plants. Plants' Fe-deficiency stress tolerance is regulated by MNB1. $M N B 1$ expression is inhibited by Fe deficiency stress, which lowers ROS levels and changes ROS-modulated signaling. This resulted in increased expression abundances of Fe-uptake and translocation genes (FIT, IRT1, FRO2, ZIF, FRD3, NAS4, PYE and MYB72), increased Fe accumulation, and enhanced Fe-deficiency tolerance (Fig.8).

\section{Accession numbers}

Sequence data from this article can be found in the Arabidopsis Genome Initiative or GeneBank / EMBL database under the following accession numbers:

MNB1 (AT1G78830), FIT(AT2G28160), FRO2(AT1G01580), IRT1 (AT4G19690), ZIF1 (AT5G13740), FRD3 (AT3G08040), NAS4 (AT1G56430), PYE (AT3G47640), MYB72 (AT1G56160) and ACTIN8 (AT1G49240).

\section{Acknowledgments}

We thank Chenchen Tong, Xiangyu Zhu,Manzhi Tao,Jiena Xu, Yangyang Han, Xue Fang,and Hongtao Wang for their technical assistance. This work was supported by the National Natural Science Foundation of China (32070276 and 31770284). The funders had no role in study design, data collection and analysis, decision to publish, or preparation of the manuscript.

\section{Author contributions}

Shuqing Cao, Li jiang and Hui Song conceived the original research plans; Hui Song, Feng Chen, Xi Wu, Min Hu, Qingliu Geng, Min Ye, Cheng Zhang performed the 
experiments; Shuqing Cao, Li jiang, Hui Song, Feng Chen and Xi Wu designed the experiments and analyzed the data; Shuqing Cao and Hui Song wrote the article with contributions of all the authors.

\section{Conflict of interest}

The authors have no conflict of interest to declare.

\section{References}

Aono, M., Kubo, A., Saji, H., Tanaka, K., Kondo, N. (1993). Enhanced tolerance to photooxidative stress of transgenic Nicotiana tabacum with high chloroplastic glutathione reductase activity. Plant Cell Physiol 34, 129-135.

Alonso JM, Stepanova AN, Leisse TJ, et al. (2003). Genome-wide insertional mutagenesis of Arabidopsis thaliana. Science $301,653-657$.

Bauer, P., Ling, H.Q., Guerinot, M.L. (2007). FIT, the FER-like iron deficiency induced transcription factor in Arabidopsis. Plant Physiol. Bioch 45, 260-261.

Balk, J., and Schaedler, T.A. (2014). Iron cofactor assembly in plants. Annu. Rev. Plant Biol 65,125-153.

Clough, S.J., Bent, A.F. (1998). Floral dip: a simplified method for Agrobacterium-mediated transformation of Arabidopsis thaliana. Plant J 16, 735-743.

Curie, C., Panaviene, Z., Loulergue, C., Dellaporta, S.L., Briat, J.F., and Walker, E.L. (2001). Maize yellow stripe1 encodes a membrane protein directly involved in Fe(III) uptake. Nature 409, 346-349.

Colangelo EP, Guerinot ML. (2004) .The essential basic helix-loop-helix protein FIT1 is required for the iron deficiency response. Plant Cell 16, 3400-3412.

Chen, J., Yang, LB., Gu, J., Bai, X.Y., Ren. Y.B., Fan, T.T., Han, Y., Jiang, L., Xiao, F.M., Liu, Y.S., Cao, S.Q. (2015). MAN3 gene regulates cadmium tolerance through the glutathione-dependent pathway in Arabidopsis thaliana. New Phytol 205, 570-582.

Cui Y, Chen CL, Cui M, Zhou WJ, Wu HL, Ling HQ. (2018). Four IVa bHLH Transcription Factors Are Novel Interactors of FIT and Mediate JA Inhibition of Iron Uptake in Arabidopsis. Mol Plant 11, 1166-1183.

Eide D, Broderius M, Fett J, Guerinot ML. (1996). A novel iron-regulated metal transporter from plants identiWed by functional expression in yeast. Proc Natl Acad Sci USA 93,5624-5628

Fan T, Yang L, Wu X, Ni J, Jiang H, Zhang Q, Fang L, Sheng Y,Ren Y, Cao S. (2016). The PSE1 gene modulates lead tolerance in Arabidopsis. J Exp Bot 67, 4685 - 4695

Garcia-Brugger A, Lamotte O, Vandelle E, Bourque S, Lecourieux D,Poinssot B, Wendehenne D, Pugin A.(2006). Early signaling events induced by elicitors of plant defenses. Mol Plant Microbe Interact 19,711-724

Hodges DM, Delong JM, Forney CF, Prange RK. (1999). Improving the thiobarbituric acid-reactivesubstances assay for estimating lipid peroxidation in plant tissues containing anthocyanin and other interfering compounds. Planta 207,604 - 611. 
Herbik, A., Bölling, C., and Buckhout, T.J. (2002). The involvement of a multicopper oxidase in iron uptake by the green algae Chlamydomonas reinhardtii. Plant Physiol 130, 2039-2048.

Hell, R., and Stephan, U.W. (2003). Iron uptake, trafficking and homeostasis in plants. Planta 216, 541551.

Hammond-Kosack KE, Parker JE.(2003).Deciphering plant-pathogen communication: fresh perspectives for molecular resistance breeding. Curr Opin Biotechnol 14, 177-193.

Hansch, R., and Mendel, R.R. (2009). Physiological functions of mineral micronutrients (Cu, $\mathrm{Zn}, \mathrm{Mn}$, Fe, Ni, Mo, B, Cl). Curr Opin Plant Biol 12, 259-266.

Hwang, I.S., Hwang, B.K. (2011). The pepper mannose-binding lectin gene CaMBL1 is required to regulate cell death and defense responses to microbial pathogens. Plant Physiol 155, 447-463.

Hindt, M.N., and Guerinot, M.L. (2012). Getting a sense for signals: regulation of the plant iron deficiency response. Biochim. Biophys. Acta 1823,1521-1530.

Han Y, Zhong Z, Song L, Olsson S, Wang Z, Lu GD. (2018). Evolutionary analysis of plant jacalinrelated lectins (JRLs) family and expression of rice JRLs in response to Magnaporthe oryzae. Journal of Integrative Agriculture 17, 1252-1266.

Jakoby M, Wang HY, Reidt W, Weisshaar B, Bauer P. (2004). FRU (BHLH029) is required for induction of iron mobilization genes in Arabidopsis thaliana. FEBS Lett 577, 528-534.

Jeong, J., Guerinot, M.L. (2009). Homing in on iron homeostasis in plants. Trends Plant Sci 14, 280 285.

Kobayashi, T., Nakanishi, H., and Nishizawa, N.K. (2010). Recent insights into iron homeostasis and their application in graminaceous crops. Proc. Jpn. Acad. Ser. B Phys. Biol. Sci 86, 900-913.

Lis H, Sharon N. (1998). Lectins: carbohydrate-specific proteins thatmediate cellular recognition. Chem Rev 98, 637-674.

Ling HQ, Bauer P, Bereczky Z, Keller B, Ganal M. (2002). The tomato fer gene encoding a bHLH protein controls iron-uptake responses in roots. Proc Natl Acad Sci USA 99,13938-13943.

Liang G, Zhang H, Li X, Ai Q, Yu D. (2017). bHLH transcription factor bHLH115 regulates iron homeostasis in Arabidopsis thaliana. J Exp Bot 68, 1743-1755.

Li X, Zhang H, Ai Q, Liang G, Yu D. (2016b). Two bHLH Transcription Factors, bHLH34 and bHLH104, Regulate Iron Homeostasis in Arabidopsis thaliana. Plant Physiol 170, 2478-2493.

Long TA, Tsukagoshi H, Busch W, Lahner, B, Salt DE, Benfey PN. (2010). The bHLH transcription factor POPEYE regulates response to iron deficiency in Arabidopsis roots. Plant Cell 22, 22192236.

Longmei, Zhai, Chaohua, Sun, Yi, \& Feng et al. (2018). Atrop6 is involved in reactive oxygen species signaling in response to iron-deficiency stress in arabidopsis thaliana. Febs Letters.

Nakagami H, Soukupová H, Schikora A, Zárský V, Hirt H. (2006). A mitogen-activated protein kinase kinase kinase mediates reactive oxygen species homeostasis in Arabidopsis. J Biol Chem $281,38697-38704$

Peumans, W.J., Van, Damme, E.J.M. (1995). Lectins as plant defense proteins.Plant Physiol 109, 347352.

Palmer CM, Hindt MN, Schmidt H, Clemens S, Guerinot ML. (2013). MYB10 and MYB72 are required for growth under iron-limiting conditions. PLoS Genet 9, e1003953.

Ruifrok AC, Johnston DA. (2001). Quantification of histochemical staining by color deconvolution. Anal Quant Cytol Histol 23, 291 - 299.

Ren, Y., Miao, M., Meng, Y., Cao, J., Fan, T., \& Yue, J., et al. (2018). Dfr1-mediated inhibition of proline 
degradation pathway regulates drought and freezing tolerance in arabidopsis. Cell Reports, 23, 3960-3974.

Suzuki N, Koussevitzky S, Mittler R, Miller G. (2012). ROS and redox signalling in the response of plants to abiotic stress. Plant Cell Environ 35,259-270

Sun,C.H., Wu, T., Zhai, L.M., Li, D.Y., Zhang, X.Z., Xu, X.F., Ma, H.Q., Wang, Y. (2016). Reactive oxygen species function to mediate the Fe deficiency response in an Fe-efficient apple genotype: an early response mechanism for enhancing reactive oxygen production. Front. in Plant Sci 7, 1049.

Toyokuni, S. (1996). Iron-induced carcinogenesis: the role of redox regulation. Free Radic. Biol. Med $20,553-566$.

Thomine S, Vert G. (2013). Iron transport in plants: better be safe than sorry. Curr Opin Plant Biol 16, 322-327.

Vert G, Grotz N, Dedaldechamp F, Gaymard F, Guerinot ML, Briat JF, Curie C. (2002). IRT1, an Arabidopsis transporter essential for iron uptake from the soil and for plant growth. Plant Cell $14,1223-1233$.

Van Damme EJM, Barre A, Rouge' P, Peumans WJ. (2004). Cytoplasmic/nuclear plant lectins: a new story. Trends Plant Sci 9, 484-489.

Van Damme, E.J.M., Lannoo, N., Peumans, W.J. (2008). Plant lectins. Adv Bot Res 48, 108-209.

Wen, C.P., Wai, J.P.M., Tsai, M.K., Yang, Y.C., Cheng, T.Y.D., Lee, M.C., Chan, H.T., Tsao, C.K., Tsai, S.P., Wu, X. (2011). Minimum amount of physical activity for reduced mortality and extended life expectancy: a prospective cohort study. Lancet 378, 1244-1253.

Wang N, Cui Y, Liu Y, Fan H, Du J, Huang Z, Yuan Y, Wu H, Ling HQ. (2013). Requirement and functional redundancy of Ib subgroup bHLH proteins for iron deficiency responses and uptake in Arabidopsis thaliana. Mol Plant 6, 503-513.

Walker, E.L., and Connolly, E.L. (2008). Time to pump iron: irondeficiency-signaling mechanisms of higher plants. Curr. Opin. Plant Biol. 11, 530-535.

Xiao, Q., Zong, Y., Lu, S. (2015). Assessment of heavy metal pollution and human health risk in urban soils of steel industrial city (Anshan), Liaoning, Northeast China. Ecotoxicol. Environ Saf 120, $377-385$.

Yi Y, Guerinot ML. (1996). Genetic evidence that induction of root Fe(III) chelate reductase activity is necessary for iron uptake under iron deficiency. Plant J 10, 835-844.

Yuan, Y.X., Zhang, J., Wang, D.W., and Ling, H.Q. (2005). AtbHLH29 of Arabidopsis thaliana is a functional ortholog of tomato FER involved in controlling iron acquisition in strategy I plants. Cell Res 15,613-621.

Yuan Y, Wu H, Wang N, Li J, Zhao W, Du J, Wang D, Ling HQ. (2008). FIT interacts with AtbHLH38 and AtbHLH39 in regulating iron uptake gene expression for iron homeostasis in Arabidopsis. Cell Res 18, 385-397.

Yan, X., Huang, Y., Song, H., Chen, F., Geng, Q., Hu, M. (2021). A MYB4-MAN3-Mannose-MNB1 signaling cascade regulates cadmium tolerance in arabidopsis. PLoS genetics, 17(6), e1009636.

Zhang J, Liu B,Li M, Feng D, Jin H, Wang P, Liu J, Xiong F, Wang J, Wang HB. (2015). The bHLH transcription factor bHLH104 interacts with IAA-LEUCINE RESISTANT3 and modulates iron homeostasis in Arabidopsis. Plant Cell 27, 787-805. 
Fig. 1. Tolerance of $m n b 1$ mutants to Fe-deficiency stress.

(A) Phenotypes of the Col-0 and mnbl plants with or without Fe. 3-day old seedlings grown on MS medium were transferred to MS (+Fe, control) or Fe-deficient (-Fe, without Fe; -Fe+Frz, with $50 \mu \mathrm{M}$ ferrozine) media for two weeks. Bar $=1 \mathrm{~cm}$. (B, C) Root length (B) and total chlorophyll contents (C) of Col-0 and mnb1 mutant plants under normal and Fe-deficiency stress conditions were measured. Values are means \pm SD from three independent biological replicates $(n=3)$. Bars marked with different lowercase letters differ significantly (Tukey's test, $p<0.05$ ).

Fig. 2. Plants overexpressing MNB1 exhibit hypersensitivity to Fe-deficiency.

(A) Phenotypes of the Col-0, OE3 and OE7 lines with or without Fe. 3-day old seedlings seedlings grown on MS medium were transferred to MS (+Fe, control) or Fe-deficient (-Fe, without $\mathrm{Fe}$; -Fe+Frz, with $50 \mu \mathrm{M}$ ferrozine) media for 2 weeks. Bar $=1 \mathrm{~cm} .(\mathrm{B}, \mathrm{C})$ Root length (B) and total chlorophyll contents (C) of Col-0, OE3 and OE7 plants under normal and Fe-deficiency stress conditions were measured. Values are means $\pm \mathrm{SD}$ from three independent biological replicates $(n=3)$. Bars marked with different lowercase letters differ significantly (Tukey's test, $p<0.05$ ).

Fig. 3. The expression patter of $M N B 1$.

(A, B) The expression of MNB1 was induced by iron-deficient stress. qRT-PCR analysis of FIT and MNB1 transcript accumulation in the roots of Col. Wild-type plants were grown on MS agar plate for 10 days and then transferred onto MS (+Fe, control) and Fe-deficient (-Fe, without Fe) for 7 days. ACTIN8 was used as the internal control. Values are means \pm SD from three independent biological replicates $(n=3)$. Bars marked with different lowercase letters differ significantly (Tukey's test, $p<0.05$ ). (C) MNB1 protein level under Fe-deficient stress. 35S:MNB1-GFP transgenic seedlings grown on MS medium for 10 days and then transferred onto MS (+Fe, control) and $\mathrm{Fe}-$ deficient (-Fe, without $\mathrm{Fe}$ ) for 7 days. Protein extracts from treated seedlings were 
analyzed by SDS-PAGE and Western blot. Anti-GFP antibody (upper panel), Rubisco (lower panel) as control.

Fig. 4. Elevated $\mathrm{H}_{2} \mathrm{O}_{2}$ and MDA concentrations in the mnbl mutants and MNB1overexpressing lines.

(A) DAB staining of Col-0, mnb1, OE3, OE7 lines. Arabidopsis seedlings grown on MS medium for a week and then transferred onto MS (+Fe, control) and Fe-deficient ($\mathrm{Fe}$, without $\mathrm{Fe}$ ) for two weeks. (B, C) Quantification of DAB staining densities in a through Image J software (B). The Measurement of MDA in Col-0, mnb1-1, mnb1-2, OE3 and OE7 plants. Arabidopsis seedlings grown on MS medium for a week and then transferred onto MS (+Fe, control) and Fe-deficient (-Fe, without Fe) for two weeks, and seedling samples were obtained and quantified in MDA concentration. Values are means \pm SD from three independent biological replicates $(n=3)$. Bars marked with different lowercase letters differ significantly (Tukey's test, $p<0.05$ ).

Fig. 5. qRT-PCR analysis of genes related to Fe-deficiency stress. (A-H) qRT-PCR analysis of genes related to Fe uptake and translocation in the roots of Col-0 and mnbl lines. The plants were grown on MS agar plate for 10 days and then transferred onto MS (+Fe, control) and Fe-deficient (-Fe, without $\mathrm{Fe})$ for 7 days. Three independent repeated experiments were conducted with similar results, each with three biological replicates. ACTIN 8 was used as the internal control. Values are means $\pm \mathrm{SD}$ from three independent biological replicates $(n=3)$. Bars marked with different lowercase letters differ significantly (Tukey's test, $p<0.05$ ). (I) IRT1 protein level under Fe-deficient stress. Col- 0 and mnbl lines grown on MS (+Fe) media for 10 days and then transferred onto MS (+Fe, control) and Fe-deficient (-Fe, without Fe) for 7 days. Protein extracts from treated seedlings were analyzed by SDS-PAGE and Western blot. Anti-IRT1 antibody (upper panel), Rubisco (lower panel) as control.

Fig. 6. The FCR activity in $m n b 1$ mutants and $M N B 1$-overexpressing plants.

(A-B) FCR activity of the Col-0, mnb1-1, mnb1-2, OE3 and OE7 plants grown on MS 
medium for a week and then transferred onto MS (+Fe, control) or Fe-deficient (-Fe) media for 3 days. The ferrozine assay was conducted on 20 pooled plant roots. Values are means \pm SD from three independent biological replicates $(n=3)$. Bars marked with different lowercase letters differ significantly (Tukey's test, $p<0.05$ ).

Fig. 7. The concentrations of Fe in different plants.

(A-D) Fe concentrations in the roots and shoots of Col-0, mnb1-1, mnb1-2, OE3, and OE7 plants under Fe-deficient stress. The plants were grown on MS agar plate for 10 days and then transferred onto MS (+Fe, control) and Fe-deficient (-Fe, without $\mathrm{Fe})$ for 7 days. Values are means \pm SD from three independent biological replicates $(n=3)$. Bars marked with different lowercase letters differ significantly (Tukey’s test, $p<0.05$ ).

Fig. 8. A model for the role of $M N B 1$ gene in modulating Fe-deficient tolerance in Arabidopsis. Fe-deficient stress inhibited the expression of $M N B 1$ gene, which leads to an increase the expression of Fe uptake and translocation gene through affecting ROS level and signaling, and thus enhanced the accumulation of Fe content in Arabidopsis.

Table S1. Primer sequences used in this study.

Fig. S1. Phenotype of mnbl mutants under many other abiotic stresses (A, C) Tolerance of Col-0, mnb1-1 and $m n b 1-2$ plants to $1000 \mu \mathrm{M} \mathrm{MnSO}_{4}, 250 \mu \mathrm{M}$ High Fe, $500 \mu \mathrm{M} \mathrm{H} \mathrm{H}_{2} \mathrm{O}_{2}$ or $1000 \mu \mathrm{M} \mathrm{H}_{2} \mathrm{O}_{2}$ stresses. 3-day old seedlings grown on $1 / 2 \mathrm{MS}$ medium were transferred to $1 / 2 \mathrm{MS}$ media with or without $1000 \mu \mathrm{M} \mathrm{MnSO}_{4}, 250 \mu \mathrm{M}$ High Fe, $500 \mu \mathrm{M} \mathrm{H}_{2} \mathrm{O}_{2}$ or $1000 \mu \mathrm{M} \mathrm{H}_{2} \mathrm{O}_{2}$ for about 2 weeks. Bar $=1 \mathrm{~cm}$. (B, D) Root length (B, D) of Col-0, mnb1-1 and mnb1-2 plants under normal and1000 $\mu \mathrm{M} \mathrm{MnSO}_{4}$, $250 \mu \mathrm{M}$ High Fe, $500 \mu \mathrm{M} \mathrm{H} \mathrm{H}_{2} \mathrm{O}_{2}$ or $1000 \mu \mathrm{M} \mathrm{H}_{2} \mathrm{O}_{2}$ stresses were measured. Values are means \pm SD from three independent biological replicates $(n=3)$. Bars marked with different lowercase letters differ significantly (Tukey's test, $p<0.05$ ). 
619 Fig. S2. Tolerance of Col-0, mnb1 mutants and MNB1-overexpressing lines to mannose 620 or Fe-deficient stress(A-B) Effect of $1.5 \mathrm{mM}$ mannose treatment on Fe-deficient 621 tolerance of the Col-0, mnb1-1, mnb1-1, OE3 and OE7 plants with or without Fe. 3-day 622 old seedlings grown on MS $(+\mathrm{Fe})$ medium were transferred to MS media with or 623 without Fe or $1.5 \mathrm{mM}$ mannose for about 2 weeks. Bar $=1 \mathrm{~cm} .(\mathrm{C}-\mathrm{F})$ Root length (C, D) 624 and total chlorophyll content (E, F) of Col-0, mnb1-1, mnb1-1, OE3 and OE7 plants 625 under normal and Fe-deficiency stress conditions were measured. Values are means \pm 626 SD from three independent biological replicates $(n=3)$. Bars marked with different 627 lowercase letters differ significantly (Tukey's test, $p<0.05$ ). 


\section{Figures}

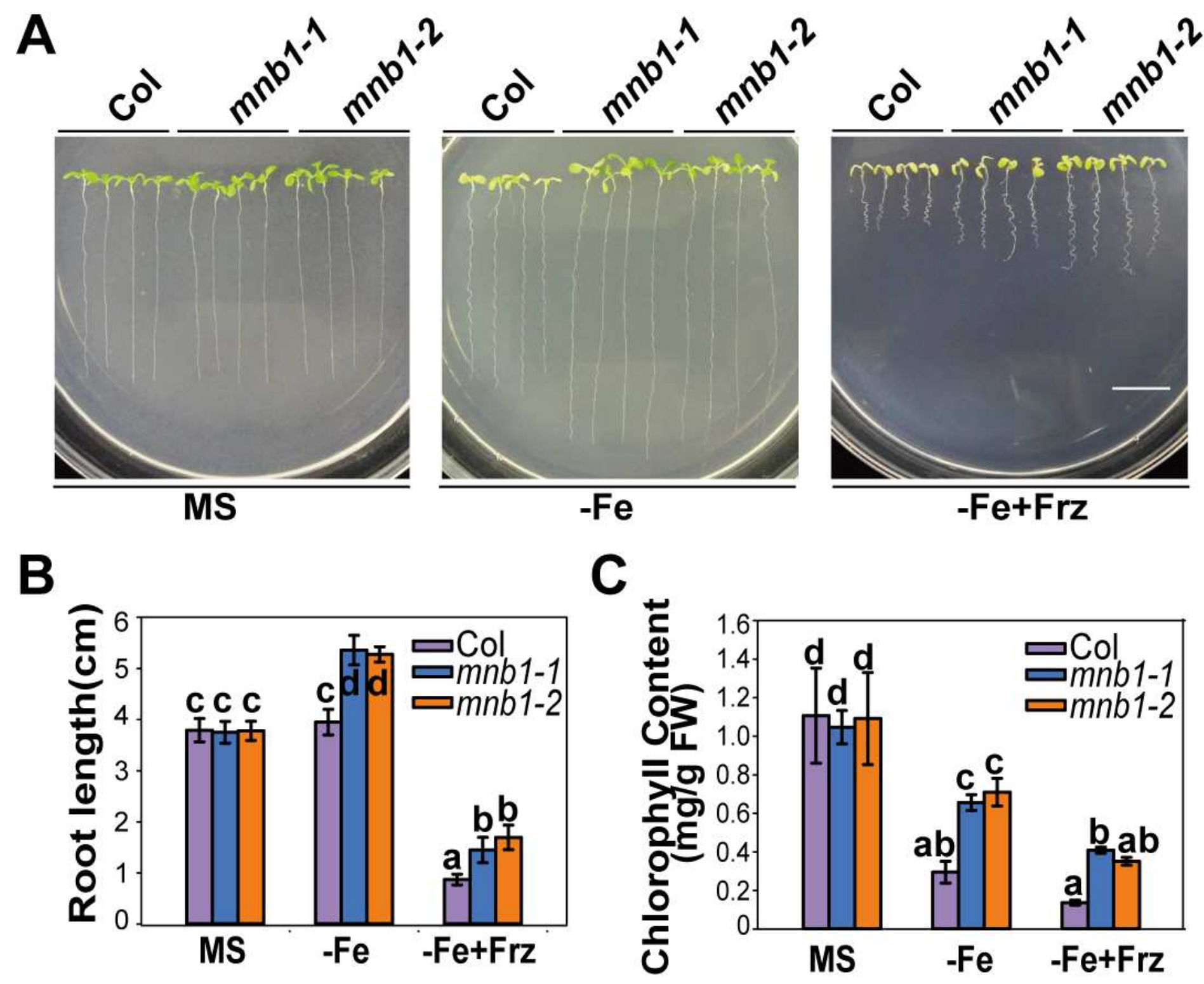

Figure 1

Tolerance of mnb1 mutants to Fe-deficiency stress. (A) Phenotypes of the Col-0 and mnb1 plants with or without Fe. 3-day old seedlings grown on MS medium were transferred to MS (+Fe, control) or Fe-deficient (-Fe, without $\mathrm{Fe}$; $-\mathrm{Fe}+\mathrm{Frz}$, with $50 \mu \mathrm{M}$ ferrozine) media for two weeks. Bar $=1 \mathrm{~cm}$. (B, C) Root length (B) and total chlorophyll contents (C) of Col-0 and mnb1 mutant plants under normal and Fe-deficiency stress conditions were measured. Values are means \pm SD from three independent biological replicates ( $\mathrm{n}$ $=3$ ). Bars marked with different lowercase letters differ significantly (Tukey's test, $p<0.05$ ). 
A

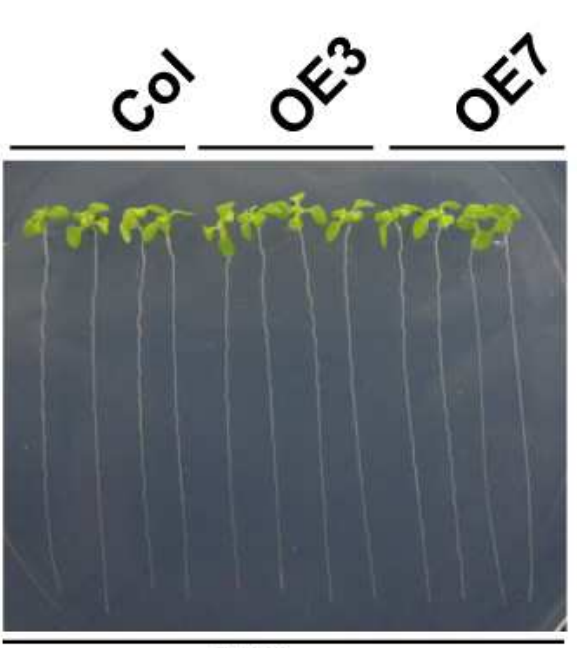

MS

B

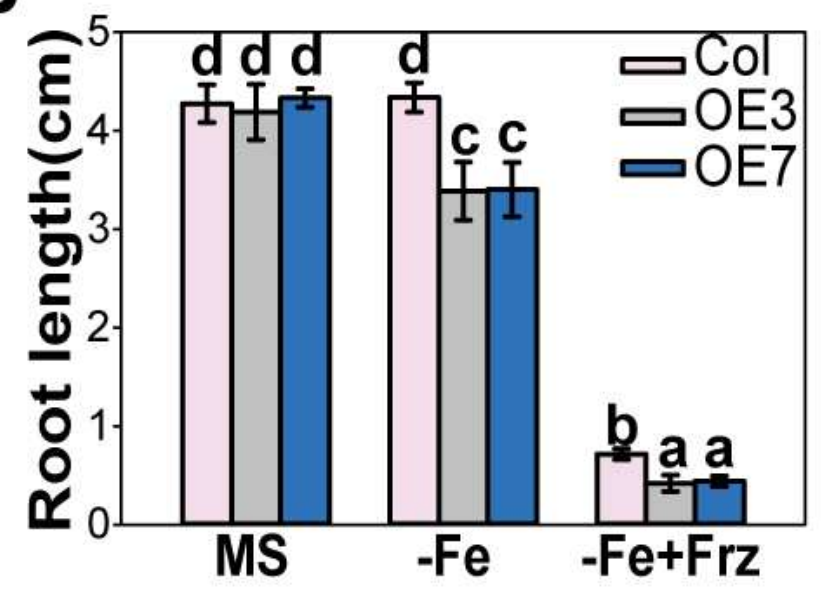

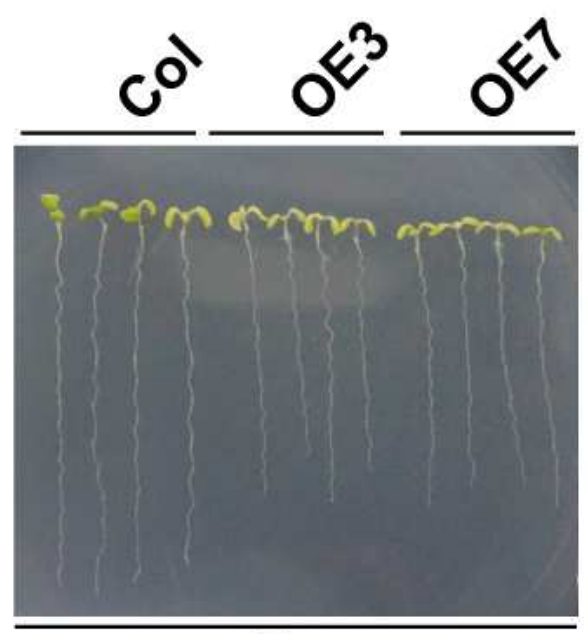

$-\mathrm{Fe}$

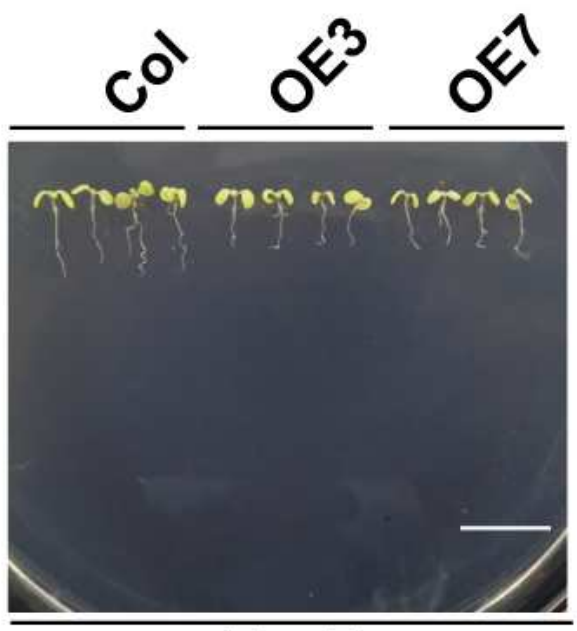

$-F e+F r z$

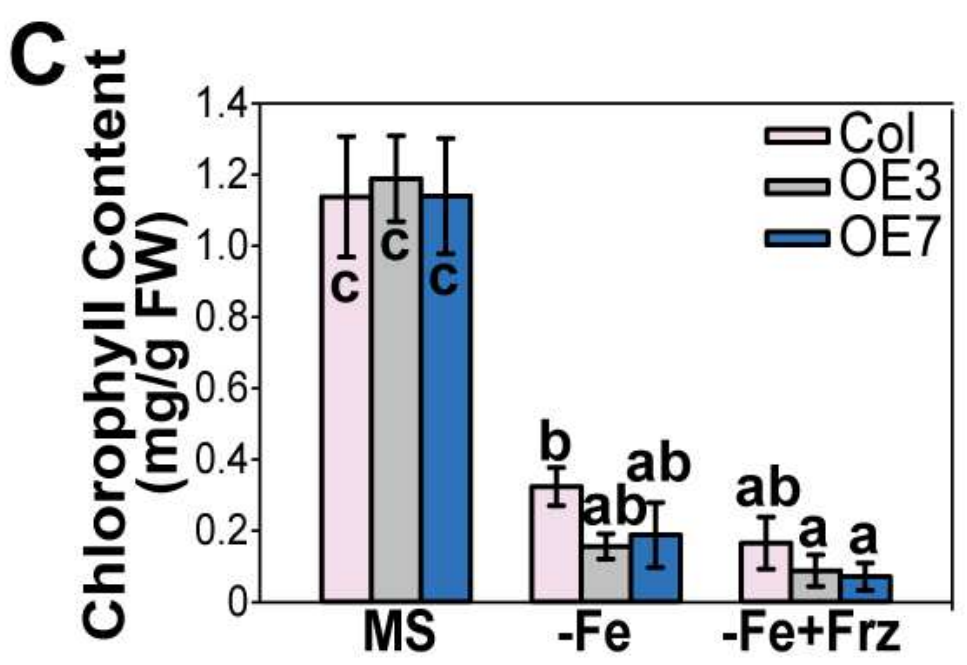

Figure 2

Plants overexpressing MNB1 exhibit hypersensitivity to Fe-deficiency. (A) Phenotypes of the Col-0, OE3 and $\mathrm{OE7}$ lines with or without Fe. 3-day old seedlings seedlings grown on MS medium were transferred to $\mathrm{MS}(+\mathrm{Fe}$, control) or Fe-deficient (-Fe, without $\mathrm{Fe}$; $-\mathrm{Fe}+\mathrm{Frz}$, with $50 \mu \mathrm{M}$ ferrozine) media for 2 weeks. Bar $=$ $1 \mathrm{~cm}$.(B, C) Root length (B) and total chlorophyll contents (C) of Col-0, OE3 and OE7 plants under normal and Fe-deficiency stress conditions were measured. Values are means \pm SD from three independent biological replicates $(n=3)$. Bars marked with different lowercase letters differ significantly (Tukey's test, $\mathrm{p}<0.05)$. 
A

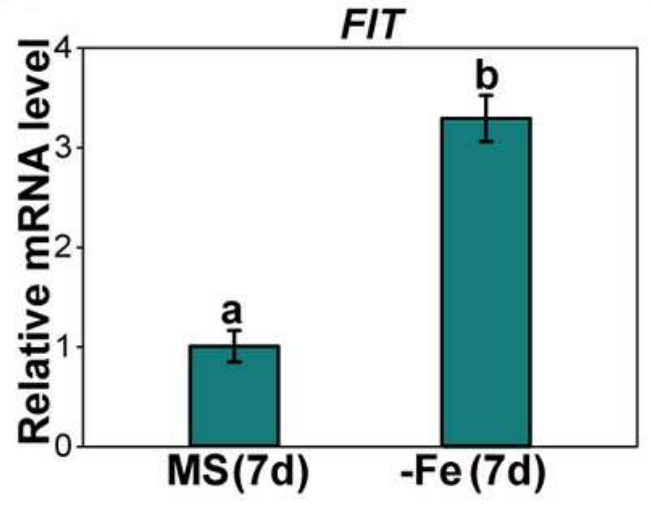

B

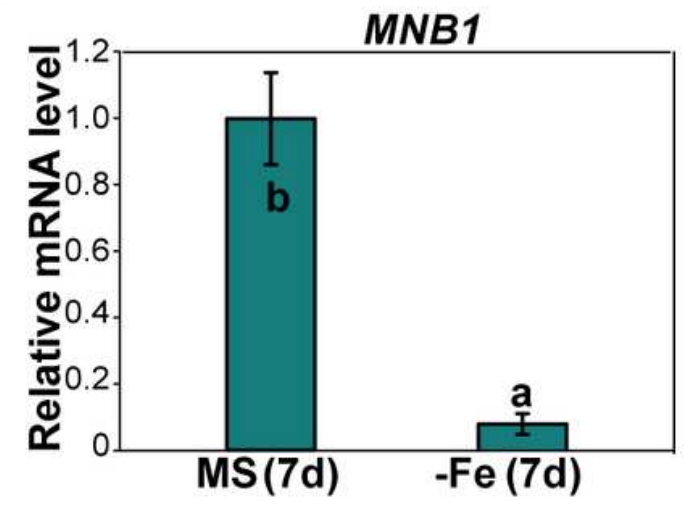

C

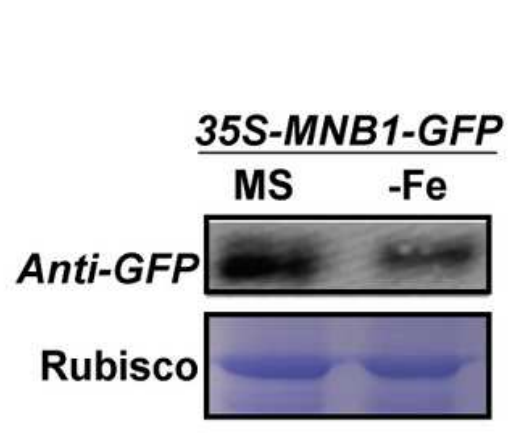

Figure 3

The expression patter of MNB1. (A, B) The expression of MNB1 was induced by iron-deficient stress. qRTPCR analysis of FIT and MNB1 transcript accumulation in the roots of Col. Wild-type plants were grown on MS agar plate for 10 days and then transferred onto MS (+Fe, control) and Fe-deficient (-Fe, without Fe) for 7 days. ACTIN8 was used as the internal control. Values are means \pm SD from three independent biological replicates $(n=3)$. Bars marked with different lowercase letters differ significantly (Tukey's test, p $<0.05)$. (C) MNB1 protein level under Fe-deficient stress. 35S:MNB1-GFP transgenic seedlings grown on MS medium for 10 days and then transferred onto MS (+Fe, control) and Fe-deficient (-Fe, without Fe) for 7 days. Protein extracts from treated seedlings were analyzed by SDS-PAGE and Western blot. Anti-GFP antibody (upper panel), Rubisco (lower panel) as control. 
A

Col

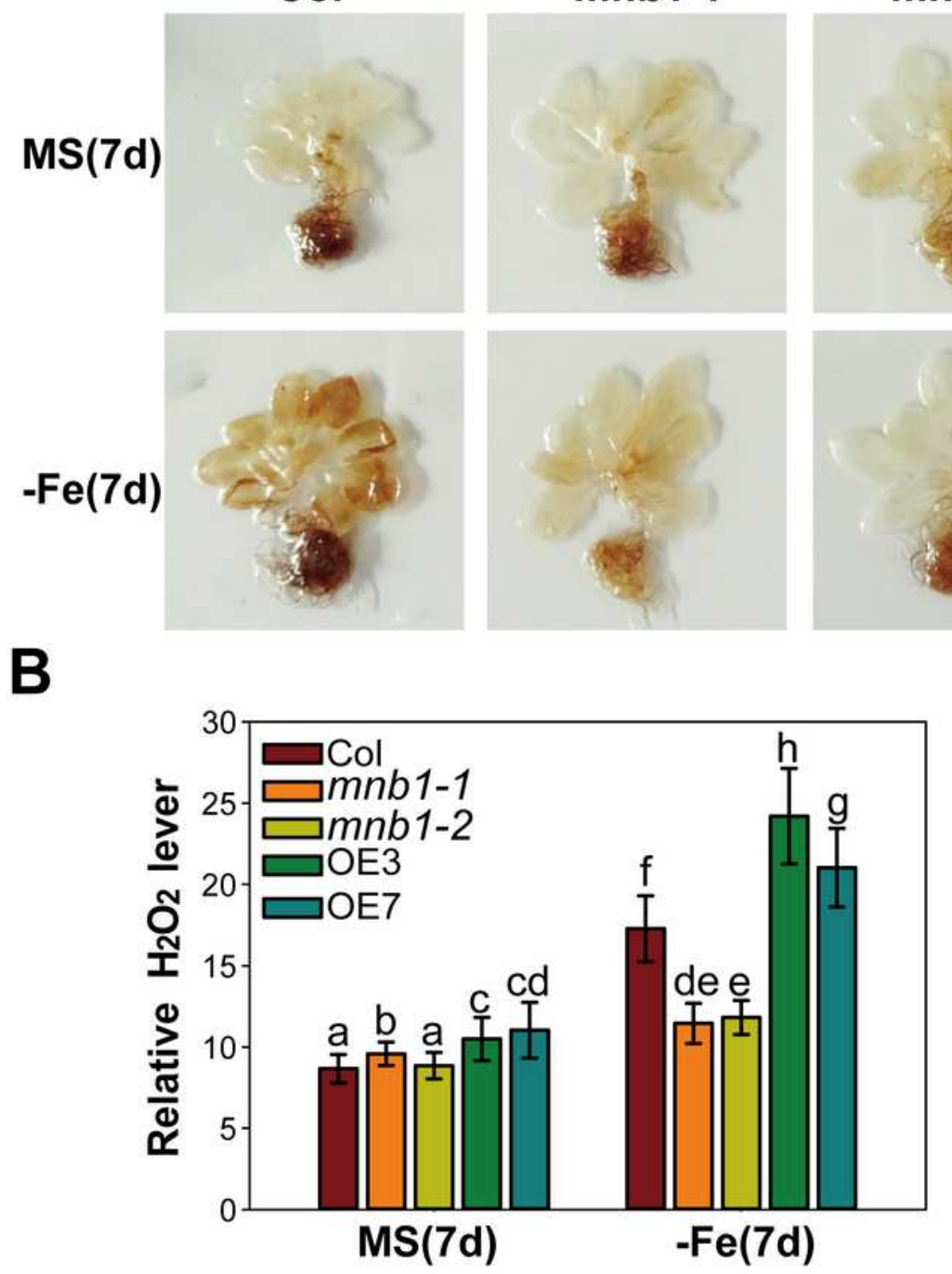

mnb1-2

OE3
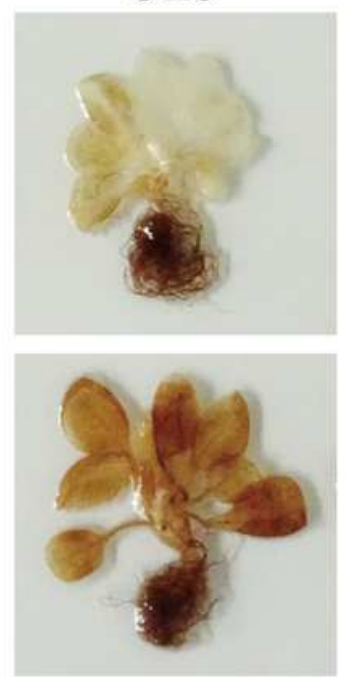

OE7
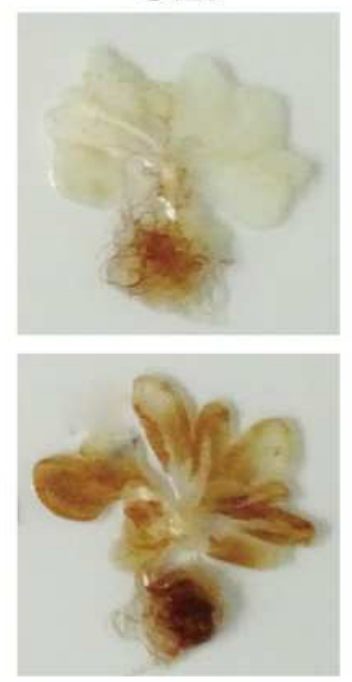

\section{C}

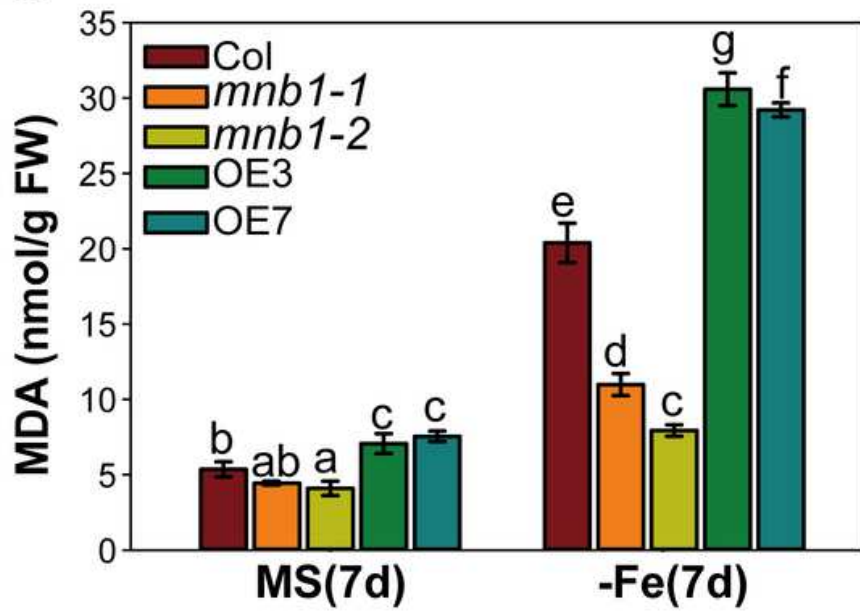

Figure 4

Elevated $\mathrm{H} 2 \mathrm{O} 2$ and MDA concentrations in the mnb1 mutants and MNB1-overexpressing lines. (A) DAB staining of Col-0, mnb1, OE3, OE7 lines. Arabidopsis seedlings grown on MS medium for a week and then transferred onto MS (+Fe, control) and Fe-deficient (-Fe, without Fe) for two weeks. (B, C) Quantification of DAB staining densities in a through Image $\mathrm{J}$ software $(\mathrm{B})$. The Measurement of MDA in Col-0, mnb1-1, mnb1-2, OE3 and OE7 plants. Arabidopsis seedlings grown on MS medium for a week and then transferred onto MS (+Fe, control) and Fe-deficient (-Fe, without Fe) for two weeks, and seedling samples were obtained and quantified in MDA concentration. Values are means \pm SD from three independent biological replicates $(n=3)$. Bars marked with different lowercase letters differ significantly (Tukey's test, $\mathrm{p}<0.05)$. 
A

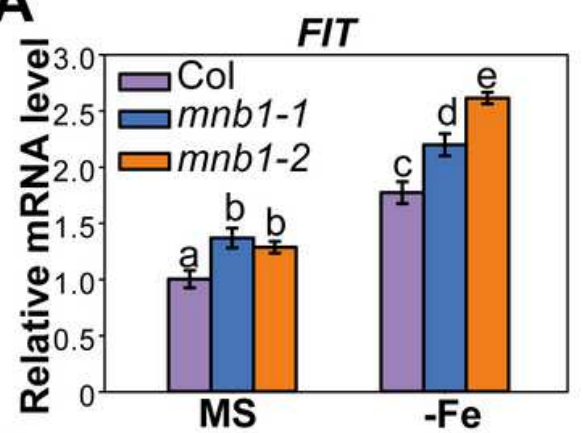

D

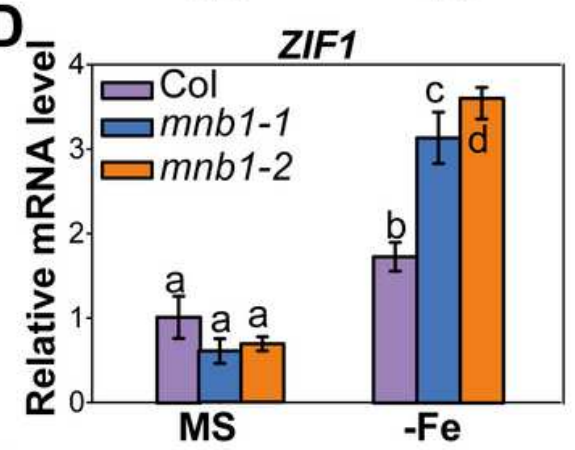

G

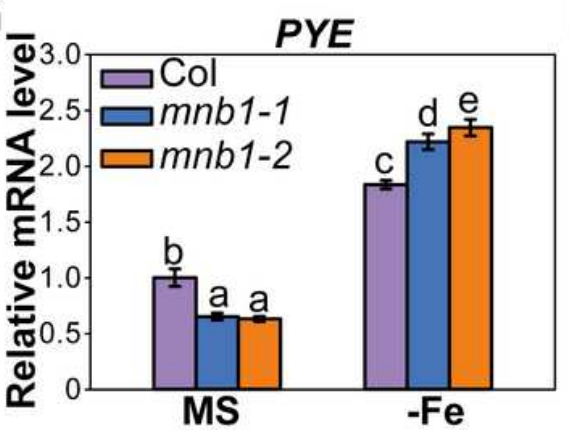

B

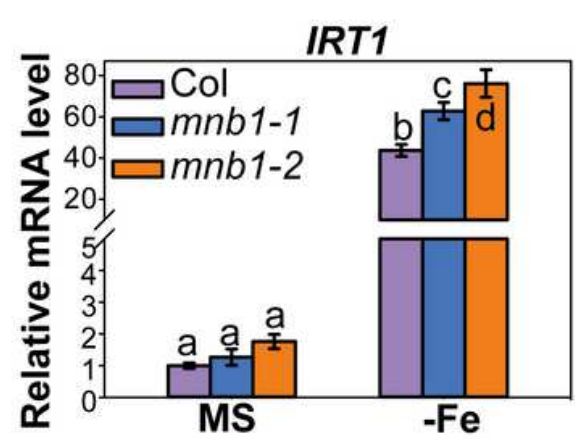

E

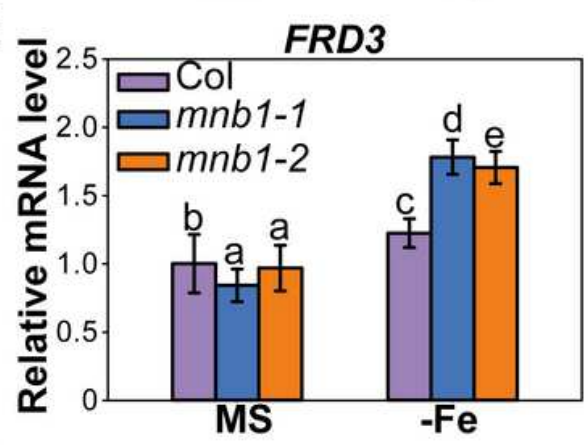

H

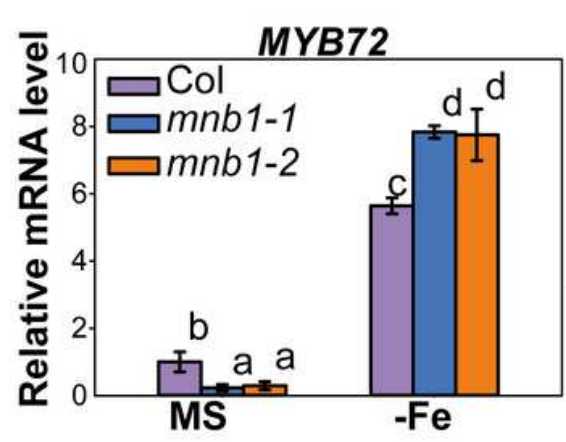

C

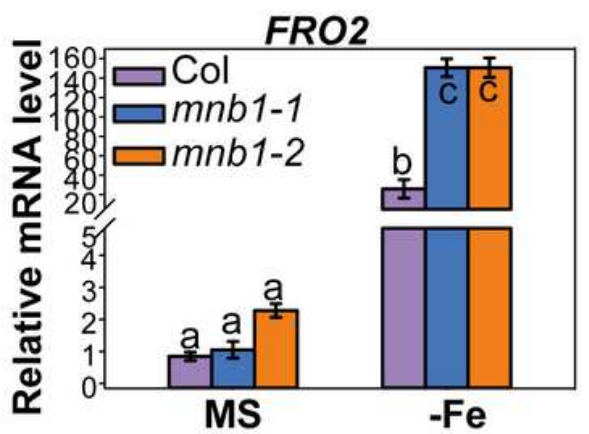

F

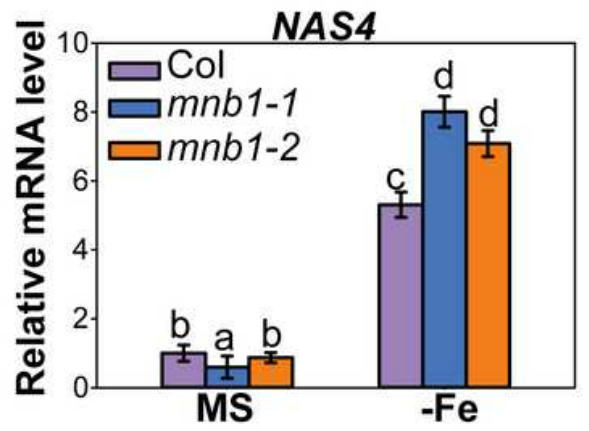

I

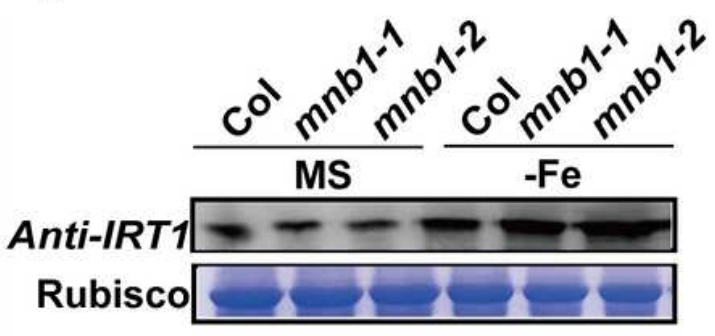

Figure 5

qRT-PCR analysis of genes related to Fe-deficiency stress. (A-H) qRT-PCR analysis of genes related to Fe uptake and translocation in the roots of Col-0 and mnb1 lines. The plants were grown on MS agar plate for 10 days and then transferred onto MS (+Fe, control) and Fe-deficient (-Fe, without Fe) for 7 days. Three independent repeated experiments were conducted with similar results, each with three biological replicates. ACTIN8 was used as the internal control. Values are means \pm SD from three independent biological replicates $(n=3)$. Bars marked with different lowercase letters differ significantly (Tukey's test, $\mathrm{p}<0.05)$. (I) IRT1 protein level under Fe-deficient stress. Col-0 and mnb1 lines grown on MS (+Fe) media for 10 days and then transferred onto MS (+Fe, control) and Fe-deficient (-Fe, without Fe) for 7 days. Protein extracts from treated seedlings were analyzed by SDS-PAGE and Western blot. Anti-IRT1 antibody (upper panel), Rubisco (lower panel) as control. 

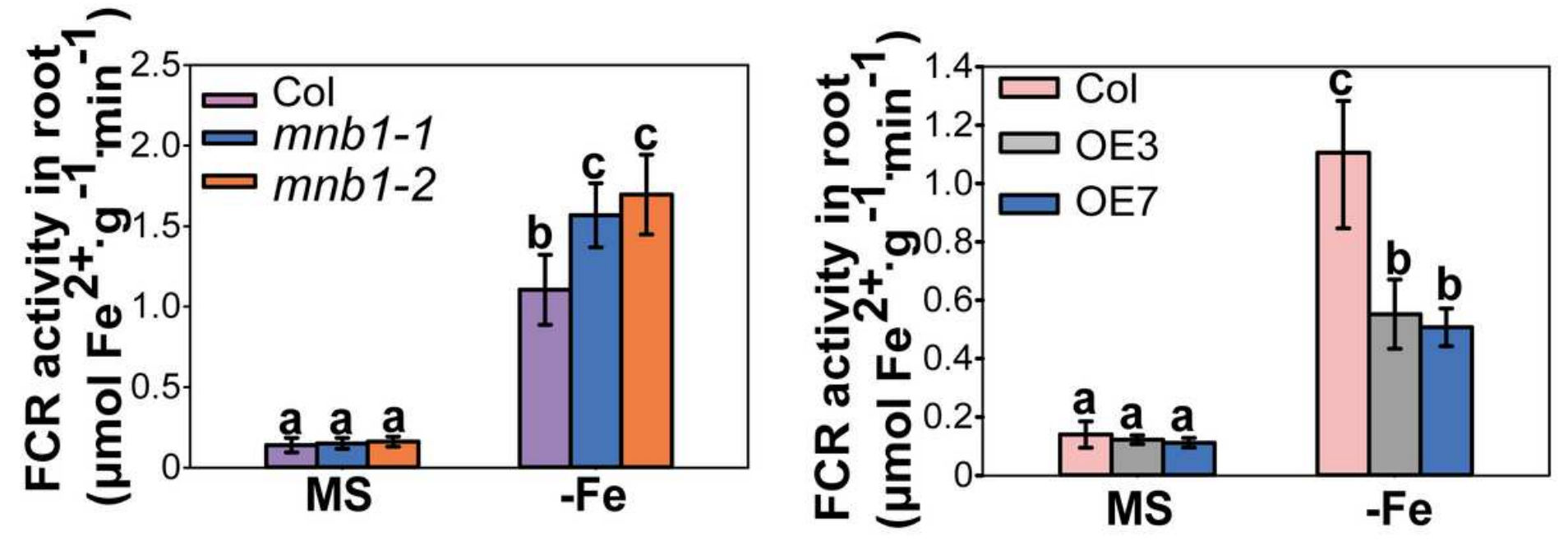

Figure 6

The FCR activity in mnb1 mutants and MNB1-overexpressing plants. (A-B) FCR activity of the Col-0, mnb1-1, mnb1-2, OE3 and OE7 plants grown on MS medium for a week and then transferred onto MS $(+\mathrm{Fe}$, control) or Fe-deficient (-Fe) media for 3 days. The ferrozine assay was conducted on 20 pooled plant roots. Values are means \pm SD from three independent biological replicates $(n=3)$. Bars marked with different lowercase letters differ significantly (Tukey's test, $p<0.05$ ). 
A
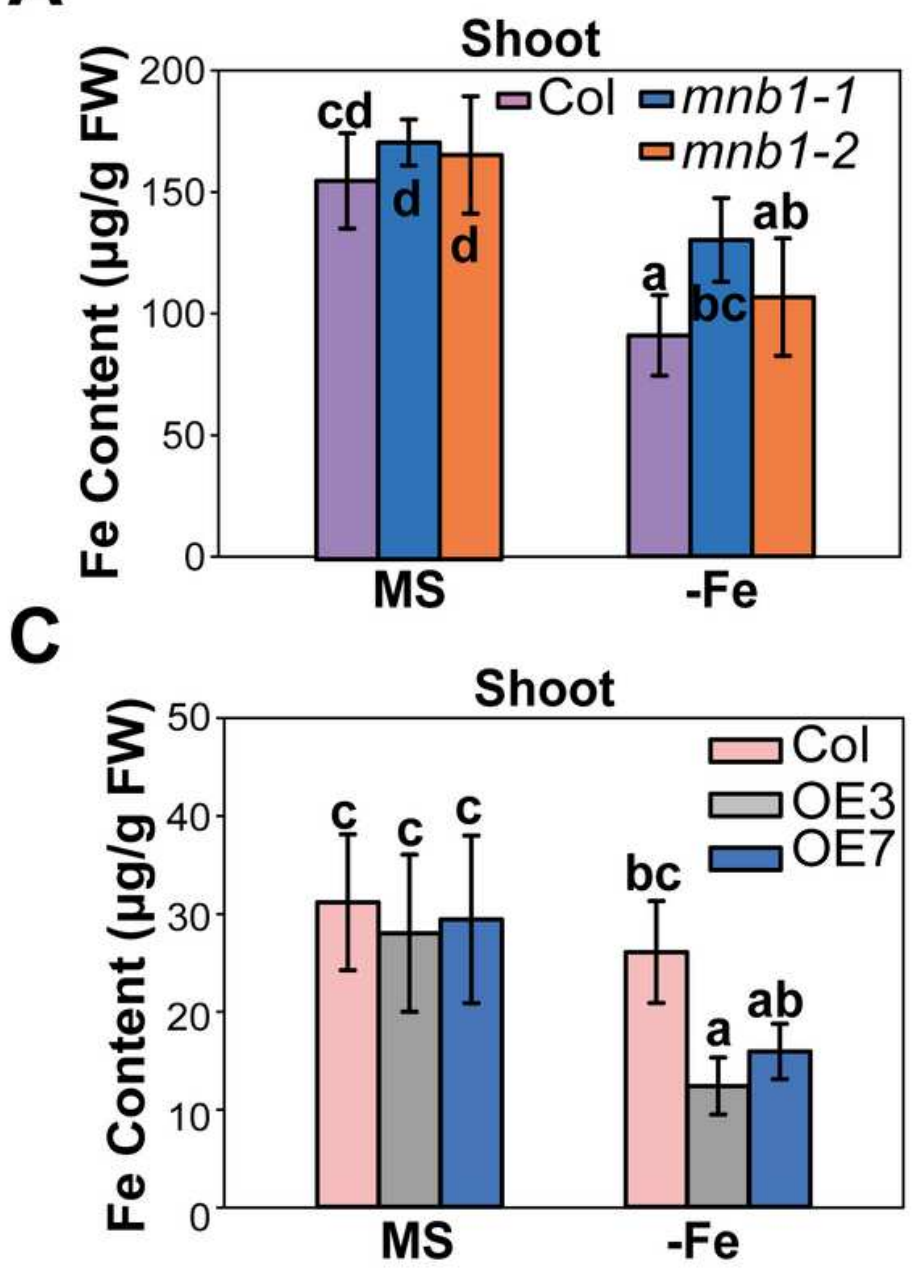

B
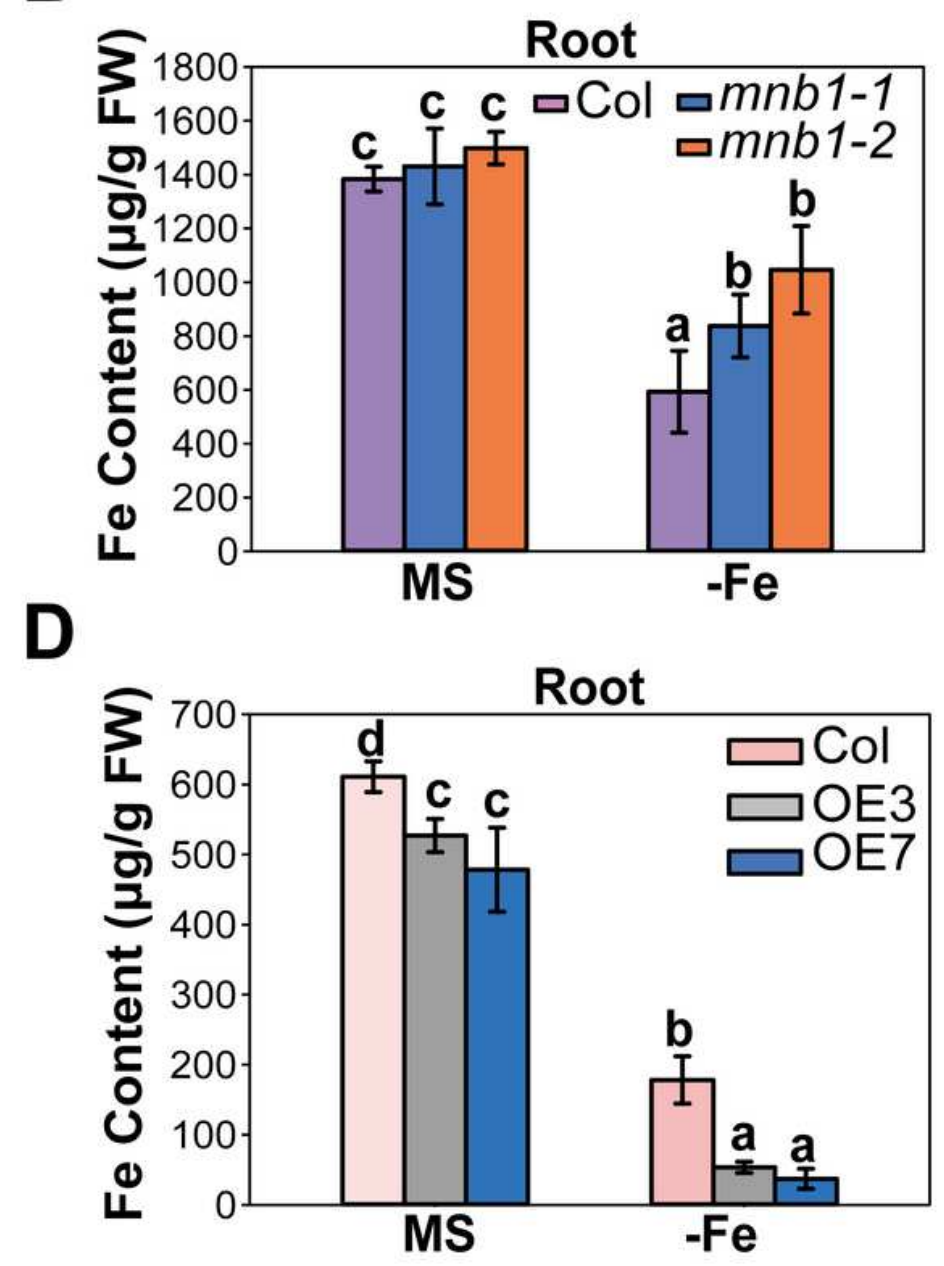

Figure 7

The concentrations of Fe in different plants. (A-D) Fe concentrations in the roots and shoots of Col-0, mnb1-1, mnb1-2, OE3, and OE7 plants under Fe-deficient stress. The plants were grown on MS agar plate for 10 days and then transferred onto MS (+Fe, control) and Fe-deficient (-Fe, without Fe) for 7 days. Values are means \pm SD from three independent biological replicates $(n=3)$. Bars marked with different lowercase letters differ significantly (Tukey's test, $p<0.05$ ). 

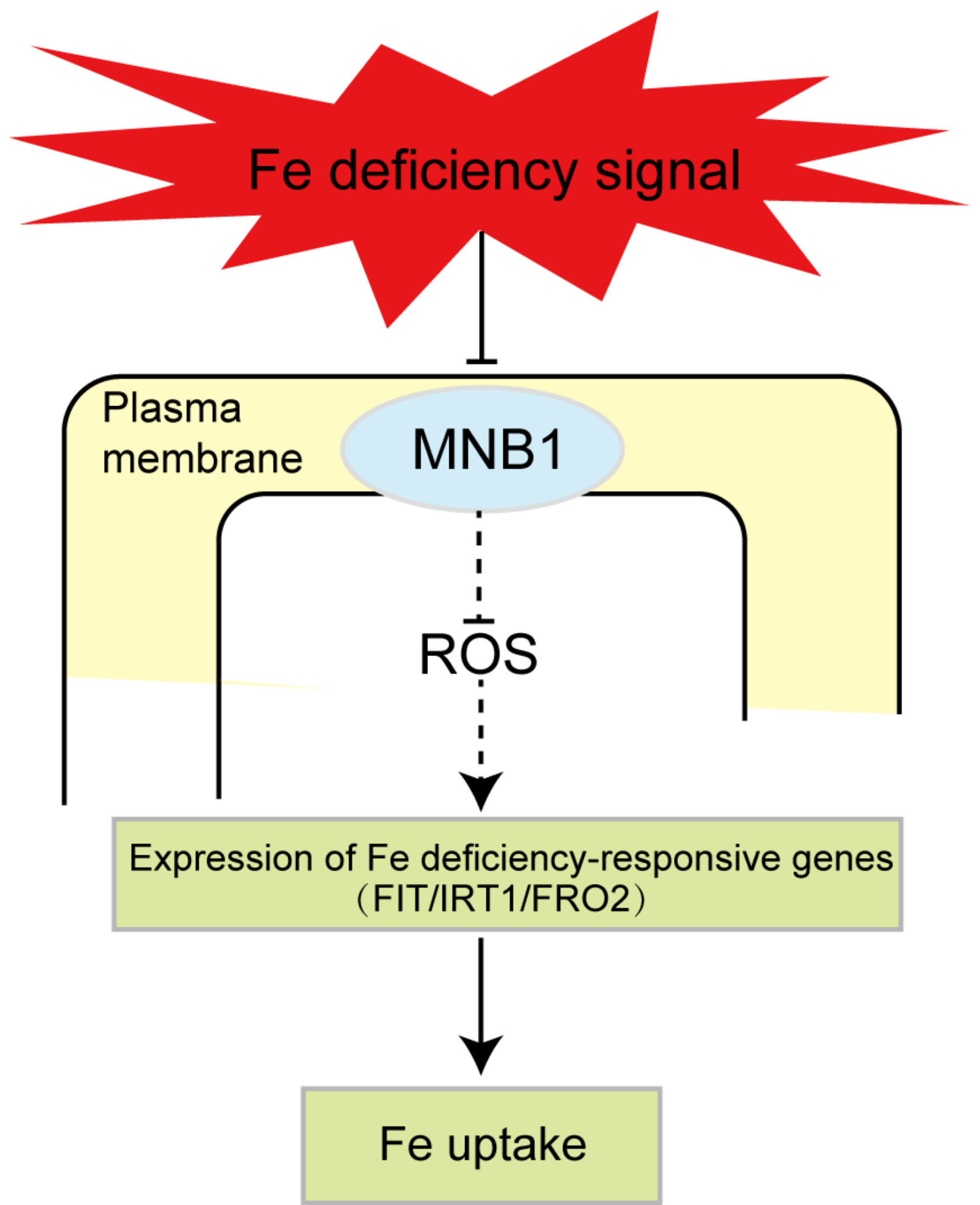

Figure 8

A model for the role of MNB1 gene in modulating Fe-deficient tolerance in Arabidopsis. Fe-deficient stress inhibited the expression of MNB1 gene, which leads to an increase the expression of Fe uptake and translocation gene through affecting ROS level and signaling, and thus enhanced the accumulation of Fe content in Arabidopsis. 


\section{Supplementary Files}

This is a list of supplementary files associated with this preprint. Click to download.

- Supplementarymaterial.pdf 